\title{
Literaturbericht
}

Elvira Rosert

\section{Fest etabliert und weiterhin lebendig: Normenforschung in den Internationalen Beziehungen}

\section{Entstehung und Entwicklung der Normenforschung in den Internationalen Beziehungen}

Ein enges normatives Netz umspannt die Welt: Verregelt und verrechtlicht sind die zwischenstaatlichen Beziehungen, in denen etwa das Gewaltverbot, Handels- und Wirtschaftsstandards oder auch diplomatische Gepflogenheiten dem Umgang der Staaten miteinander einen Handlungsrahmen geben. Auch im Umgang mit Individuen sind staatlicher Willkür durch Menschenrechte und humanitärvölkerrechtliche Bestimmungen Grenzen gesetzt.

Empirisch erfasst und theoretisch eingebettet werden diese normativen Strukturen von der Normenforschung, die sich in den Internationalen Beziehungen (IB) inzwischen als ein innovativer und dichter Diskussionszusammenhang etabliert hat. Dass erste Arbeiten zur Bedeutung von Normen in der zweiten Hälfte der 1980er Jahre entstanden und dass diese Forschungsrichtung in den neunziger Jahren enorm an Fahrt gewinnen konnte, kann als zwei miteinander verwobenen Dynamiken geschuldet angesehen werden: der weltpolitischen und der wissenschaftspolitischen. Zuvor wurde der Konflikt zwischen den Supermächten vom neorealistischen Pessimismus hinsichtlich der Frage, ob es jemals gelingen könnte, die bipolare Konfrontation zu überwinden, begleitet (Wohlforth 1994: 91). Noch vor der konstruktivistischen Welle zeigte sich jedoch in der Neo-Neo-Debatte, dass zumindest einige Wissenschaftler Kooperation statt Konfrontation nicht nur für politisch wünschenswert, sondern auch für theoretisch begründbar hielten (Müller 2013: 613 ff.). Indes deutete sich 1985 mit dem Amtsantritt Michail Gorbatschows die Möglichkeit einer tatsächlichen Veränderung an, und die 1990er Jahre wurden nach Ende des OstWest-Konflikts euphorisch zum Menschenrechtsjahrzehnt ausgerufen. Der von diesen weltpolitischen Entwicklungen getragene Wunsch, Perspektiven für weiteren 
Fortschritt aufzuzeigen, begründete die Normenforschung als neuen Forschungsstrang - dieser versprach alternative Konzeptionen des internationalen Systems und seiner Akteure und verschrieb sich dem Wandel (Sikkink 2008). In seiner ersten Entwicklungsphase ${ }^{1}$ galt es daher, nachzuweisen, dass Normen eine Rolle spielen und damit den Skeptikern den Wind aus den Segeln zu nehmen. In der zweiten Phase stand im Fokus, wie Normen ihre Wirkung entfalten, und in der dritten, warum sie in verschiedenen Kontexten und auf verschiedene Akteure unterschiedlich wirken (Finnemore/Sikkink 2001: $396 \mathrm{f}$.). Auch der Normgenese wandte man sich zu, nachdem deutlich wurde, dass die Regimeforschung wenig Aufschluss über die Phänomene geben konnte, die jetzt von Interesse waren: Ging es zuvor primär um die Lösung zwischenstaatlicher Kooperationsprobleme, stand nun die Frage nach der Herausbildung normativer Ansprüche im Vordergrund. Die aktuelle Phase ist durch Vielfalt und ein sicheres konzeptionelles Fundament gekennzeichnet, welches differenzierte Perspektiven auf die Entstehung (Santa-Cruz 2005; Carpenter 2007 a; Bailey 2008), Diffusion und Wirkung ${ }^{2}$ sowie Durchsetzung von Normen ${ }^{3}$ ermöglicht. Außerdem lenken neue Arbeiten den Blick auf bisher vernachlässigte Entwicklungen wie die Konkurrenz zwischen Normen, ${ }^{4}$ von ihnen ausgehende nicht-intendierte Effekte (Tannenwald 2007; Rosert 2011), die grundsätzliche Umstrittenheit von Normen (Wiener 2004; Wiener 2009) oder sogar deren Erosion (Rosert/Schirmbeck 2007; McKeown 2009).

Angesichts der Größe und disziplinären Anerkennung der Normenforschung ist die Notwendigkeit, ihren Mehrwert gegenüber etablierten Ansätzen zu verteidigen, merklich gesunken. Die Abgrenzung von dominanten Paradigmen ist jedoch weiterhin identitätsstiftend (Reus-Smit/Snidal 2008: 12, 32). So bleibt der (Neo-)Realismus all dies, was die Normenforschung nicht ist oder zumindest nicht sein wollte: pessimistisch, individualistisch und dennoch zu strukturdeterministisch, materialistisch und positivistisch. Am Neoinstitutionalismus reibt man sich zwar mit weniger Inbrunst, ist aber dennoch stets bestrebt, dessen Verständnis von Normen als rationalistisch verengt zu kritisieren. Das Abarbeiten an diesen paradigmatischen Lieblingsfeinden war zweifellos äußerst fruchtbar - zugleich sind nicht zuletzt deshalb einige blinde Flecken entstanden. Dieser Beitrag hat zum Ziel, zentrale theoretische Konzepte und Erträge der Normenforschung darzustellen und solche blinden Flecken und sich daraus ergebende Forschungsdesiderate herauszuarbeiten.

1 Die Einteilung in Phasen ist nicht strikt zu verstehen, sondern gibt die dominanten Forschungsschwerpunkte wieder.

2 Kelley 2008; Greenhill 2010; Hyde 2011; Zwingel 2012.

3 De Nevers 2007; Hafner-Burton 2008; Donno 2010, Risse/Sikkink/Ropp 2013.

4 Carpenter 2007 b; Sandholtz 2008; Elgström 2009; Cho 2012; Moore 2012. 
Er soll als Anregung dienen, das nach wie vor reichlich vorhandene Erkenntnispotenzial in drei Richtungen auszubauen: Systematisierung, (konditionale) Generalisierung und kontraintuitive Perspektiven auf Normen.

\section{Normen als Konzept: Definitionen, Charakteristika und Funktionen}

Nach der langen Uneinigkeit darüber, ob Normen normativ als Verhaltensvorschriften (Krasner 1983: 2) oder behavioristisch als Verhaltensregelmäßigkeiten (Axelrod 1986: 1097; Thomson 1993: 80) zu konzipieren sind, ist in der konstruktivistischen Literatur schließlich eine Definition von Normen als ,collective expectations about proper behavior for a given identity“ (Jepperson/Wendt/Katzenstein 1996: 54) zum Standard avanciert. Dieses Verständnis vereint sowohl die Normativität als auch die Normalität von Normen: Sie sind als Gebote oder Verbote formulierte Handlungsanweisungen (Tannenwald 1999: 436; Hurrell/Macdonald 2013: 69-70; Deitelhoff 2006: 37); damit einhergehende Praktiken erlauben wiederum den Rückschluss auf die zugrunde liegende Norm (Onuf 1998: 59). Jenseits dieses Konsenses unterscheiden sich die Auffassungen darüber, welche Eigenschaften von Normen essenziell und welche zwar häufig, jedoch nicht notwendig sind. Müssen alle Normen moralischen Gehalt haben (Nadelmann 1990; Goertz/ Diehl 1992) oder können sie auch bloß funktional sein (Klotz 1996: 14; Bull 1977: 53)? Müssen alle Normen mit Sanktionsaussichten verbunden sein (Goertz/Diehl 1992: 638) oder nicht (Katzenstein 1996: 21)? Muss das Abweichen von der Norm tatsächlich bestraft werden (Axelrod 1986: 1097) oder reicht bereits dessen verbale Missbilligung aus (Lutz/Sikkink 2000: 655)?

Normen erfüllen eine Reihe von Funktionen für den einzelnen Akteur wie auch für die gesellschaftliche Ordnung. Durch ihren imperativen Charakter fungieren sie als Orientierungspunkt und Verhaltensmaßstab, das heißt, dass sie das erwartete Verhalten für „wiederkehrende soziale Handlungssituationen“ (Deitelhoff 2006: 37) spezifizieren und dadurch definieren, welches Verhalten als ordnungsgemäß zu werten ist (Bull 1977: 53). Damit einher geht eine Ordnungs- und Kommunikationsfunktion, wenn Normen eine soziale Ordnung schaffen, innerhalb dieser als interpretativer Rahmen dienen und zur Problemlösung beitragen (March/Olsen 1989: 52; Kratochwil 1991: 69 f.). Während diese Funktionen vermutlich allen Normen inhärent sind, ist bei den von Konstruktivisten hervorgehobenen Meta-Funktionen - Konstitution von Identität, Inklusion und Exklusion (Legro 1997: 54; Elster 1989: 109) - offenkundig, dass sie nicht von jeder Norm in gleicher Weise (und notwendigerweise) erbracht werden. 
Diese differenzierte Konzeptualisierung sollte stärker systematisiert werden, indem aus unterschiedlichen Eigenschaften und Funktionen Normtypen gebildet und mögliche Zusammenhänge untersucht werden: Entstehen moralische Normen anders als nicht-moralische und welche werden eher eingehalten? Hängen die Sanktionsart und die Sanktionsbereitschaft mit der Funktionalität und dem moralischen Anspruch der Norm zusammen? Sind Normen, die bestimmte Funktionen erfüllen, effektiver als andere? Warum werden einige Normen zu Identitätsmerkmalen, andere nicht?

\section{Die Entstehung und Verbreitung von Normen: Ursprünge, Akteure und Mechanismen}

Wenn von Normentstehung die Rede ist, interessieren sich die Autoren in der Regel für die Genese und Diffusion von Normen. Mit Bezug auf internationale Verhandlungen definiert Nicole Deitelhoff (2006: 45) Normgenese als den Prozess, ,durch den sich ein neuer normativer Anspruch innerhalb einer Gruppe von Akteuren bildet". Überträgt sich die vereinbarte Norm auf andere Akteure und erfährt sie zunehmende internationale Akzeptanz, spricht man von Normdiffusion (Finnemore/ Sikkink 1998: 902; Santa-Cruz 2005: 671; Checkel 1999: 85). Während sich die meisten Arbeiten Prozessen gezielter Normsetzung widmen, in denen Verhaltensstandards gezielt aus der Normativität entstehen, weisen andere Autoren in die Gegenrichtung: Normativität folge unter Umständen erst der Normalität, wenn sich Normen aus der Praxis herausbilden - dabei werden Verhaltensregelmäßigkeiten zu Verhaltensmaßstäben mit Verpflichtungscharakter, weil sie Erwartungen generieren (Ullmann-Margalit 1977: 11; Krasner 1983: 18 f.; Raymond 1997: 217). Dieses konzeptionellen Hinweises ungeachtet befassen sich allerdings nur wenige Studien empirisch mit der spontanen Herausbildung von internationalen Normen (Kamis 2011), sodass dieses Phänomen wie auch die Frage, ob gezielt geschaffene oder zufällig entstandene Normen langlebiger und effektiver sind, Forschungsdesiderate darstellen. Ferner bietet der Mechanismus der Selektion, den Ann Florini (1996) in ihrer evolutionstheoretisch inspirierten Perspektive beschreibt, einen Ansatzpunkt, um die wenig erforschte Konkurrenz unter Normen in den Blick zu nehmen.

\section{a) Normunternehmer: Akteure und Motive}

Da aus konstruktivistischer Sicht die Entstehung von Normen eines kollektiven Problembewusstseins bedarf, welches gegebenenfalls erst in Aushandlungsprozessen hergestellt werden muss, sind norm entrepreneurs, die ebendies tun, zu einem wichtigen Forschungsfokus geworden. Letzterer ist allerdings dreifach verengt: 
erstens im Hinblick auf die Frage, wann, das heißt in welchen Phasen der Normentwicklung Normunternehmer aktiv sind; zweitens, wer, das heißt welche Akteure als Normunternehmer (und respektive als Normadressaten) aktiv sind; und drittens, warum, das heißt aus welchen Motiven heraus Normunternehmer handeln.

Die meisten Studien ${ }^{5}$ konzentrieren sich auf das Stadium der Normgenese und zeigen die Strategien auf, mit denen Normunternehmer versuchen, die Präferenzen der Normadressaten zu verändern. Hier wäre eine begriffliche wie auch konzeptionelle Erweiterung um diejenigen Akteure wünschenswert, die sich, etwa durch Monitoring, auch nach der Entstehungsphase für die Norm engagieren (Wunderlich 2013: 35 f.) - sie werden bislang typischerweise nicht als Normunternehmer bezeichnet (bspw. Forschungsgruppe Menschenrechte 1998).

Nach Margret Kecks und Kathryn Sikkinks (1998) bahnbrechendem Werk über Aktivisten-Netzwerke (transnational advocacy networks, TANs) und befördert durch den generellen Boom der NGO-Forschung in den 1990er Jahren, sind lange Zeit primär nicht-staatliche Akteure - neben NGOs auch Wissensgemeinschaften (epistemic communities; einschlägig Haas 1989) - als Normunternehmer erforscht worden. Einige Studien haben das Potenzial internationaler Organisationen zur Normunternehmerschaft aufgezeigt. ${ }^{6}$ Dass sich diese Akteursgruppen als fähig erwiesen, ihr Defizit an traditionellen Machtressourcen mit diskursiven Ressourcen wie Glaubwürdigkeit, Expertise und Argumenten (Deitelhoff 2009: 44) zu kompensieren und fulminante Verhandlungserfolge zu erzielen, hat die Grenzen der rationalistischen und neorealistischen Ansätze demonstriert. Zugleich erklärt dieser Fokus, weshalb die Normenforschung die Normunternehmerschaft von Staaten und auch da zunächst vor allem die kleinerer und mittlerer Mächte (Ingebritsen 2002; Rutherford/Brem/Matthew 2003) unter Ausblendung der Großmächte - erst später entdeckte: 7 Schließlich verfügen (mächtige) Staaten über genügend klassische Druckmittel, um ihren Peers Normen schlicht aufzuzwingen (De Nevers 2007: 56), womit sie für die an der Macht der Überzeugung interessierten Konstruktivisten uninteressant waren. Begünstigt wurde diese Vernachlässigung sicherlich auch durch die Tatsache, dass sich die meisten Normen an Staaten richten und diese deshalb vorwiegend als Normadressaten - und eben nicht als Normunternehmer wahrgenommen werden. Löst man den Dualismus ,,nicht-staatlicher oder zwischenstaatlicher Normunternehmer - staatlicher Normadressat" auf, wird das Akteursspektrum in beiden Rollen breiter: Auch Unternehmen treten nun als Normadres-

5 Beispielsweise Nadelmann 1990, Price 1998 oder Clark 2001.

6 Etwa Finnemore 1993, Santa-Cruz 2005, Bearce/Bondanella 2007 oder auch Greenhill 2010.

7 Eine frühe Ausnahme bilden Kaufmann/Pape 1999. 
saten und als Normunternehmer in Erscheinung (Hofferberth u. a. 2011; Flohr u. a. 2010); internationale Organisationen (Capie 2008) und nicht-staatliche Akteure (Herr 2010) werden auch als Normadressaten interessant.

Diesen Erweiterungen muss auch ein genauer Blick auf die motivationalen und strukturellen Veränderungen innerhalb der Normunternehmer-Gemeinschaft folgen. Gemeinhin wird angenommen, dass sich Normunternehmer vor allem für die Anliegen von Dritten engagieren und damit aus altruistischen, emphatischen und ideellen Motiven handeln (Finnemore/Sikkink 1998: 912). Doch was bedeutet es für die Entstehung von Normen, wenn, wie in der Global-Governance-Forschung bereits diskutiert, die Professionalisierung und Vergrößerung des NGO-Sektors zu ungleicher Machtverteilung, Ressourcenkonkurrenz und Kompetenzgerangel führt (Cooley/Ron 2002) oder sich Netzwerke mit konkurrierenden Zielsetzungen bilden (Bailey 2008: 303)? Wie einige Studien zeigen, sind TANs außerdem in der Regel nicht allein tätig, sondern agieren zusammen mit staatlichen Verbündeten in sogenannten ,like-minded“-Koalitionen (Kelley 2008: 226). Beeinflussen diese Entwicklungen die Wahl der ,issues"? Wie entscheiden Normunternehmer, welche Probleme sie in die Öffentlichkeit tragen - und welche nicht? Ist die Dringlichkeit - oder die Vermarktbarkeit - eines Problems maßgeblich? Sind Normen, die aus „like-minded“-Prozessen hervorgehen, schwächer (weil etwa der Zwang zur Einigung zur Aufgabe radikaler Positionen führt) oder, im Gegenteil, stärker (weil radikale Positionen in solchen Koalitionen mehr Gehör finden als außerhalb)? Erste Studien, die die Entstehung und Nicht-Entstehung bestimmter Normen mit diesen Faktoren erklären, sind bereits erschienen (Carpenter 2007 a; 2011) oder in Arbeit (Sigman/Schmitz/Mergel 2011). Es bleibt aber noch viel Erkenntnispotenzial. Gleiches gilt für die Motive, aus denen heraus Staaten aktiv werden: Hier wäre eine stärkere Überprüfung der Interessenshypothese wünschenswert, denn es gibt Hinweise darauf, dass die ,good international citizens“ in Bereichen mit eigenen Interessen normablehnendes Verhalten hinter der sonst guten Reputation verstecken (Bailey 2008: 305 f.).

\section{b) Strategien: Framing, sozialer Druck und Überzeugung}

Eine viel erforschte Strategie, welche Normunternehmer zur Vermittlung neuer Angemessenheitsstandards einsetzen, ist das ,Framing“, das Einbetten von Problemen in interpretative und normative Strukturen. ${ }^{8}$ Vier Eigenschaften gelten als entscheidend für seine Effektivität: die Entnormalisierung der bestehenden Praxis, die

8 Siehe hierzu etwa Finnemore/Sikkink 1998: 908, Keck/Sikkink 1998: 10, 19, Price 1998: 628 ff. sowie Payne 2001: 38. 
Emotionalisierung und Dramatisierung des Sachverhaltes, die Deutlichkeit und Einfachheit der Botschaft und die Anschlussfähigkeit an bestehende Normen (grafting). Spannend ist hier die Frage, ob sich jeder Sachverhalt - unter Anwendung der genannten Strategien - beliebig einrahmen lässt und wenn nicht, wo die Grenzen der „Rahmbarkeit“ liegen; außerdem ist zu überprüfen, ob diejenigen Frames, die immer wieder als Erklärungsfaktoren für erfolgreiche Normsetzungsprozesse identifiziert werden, in allen Erfolgsfällen vorhanden waren und damit als notwendige Bedingungen gelten können. In Fällen von Misserfolg ist zu klären, welche weiteren Bedingungen gefehlt haben, sodass die Normbildung letztlich scheiterte. Des Weiteren könnte die Wirkung bestimmter Frames stärker (und kritischer!) in den Blick genommen werden: Welche Bilder werden damit reproduziert, welchen Preis haben solche strategisch gewählten Verzerrungen und Vereinfachungen? Wie verändert sich die Empfänglichkeit der Öffentlichkeit - verfangen etwa Probleme, die sich nicht in dominante Frames fassen oder auf einfache Botschaften reduzieren lassen, weniger, weil sich die Öffentlichkeit an bestimmte Auslöser gewöhnt hat und durch andere nicht (mehr) aktivieren lässt?

Framing zielt nicht nur auf die Vermarktung der ,issues“, sondern auch auf den Aufbau sozialen Drucks: Das Stichwort-Trio „naming, blaming, shaming“ meint das „Anprangern staatlichen Fehlverhaltens“, welches die Adressaten unter einen „Rechtfertigungs- und Anpassungsdruck“ setzt (Liese 2006: 104). Empirische Beispiele (aktuell etwa Syrien) wie auch einzelne Arbeiten (Hafner-Burton 2008) demonstrieren jedoch die Widerstandskraft einiger Akteure gegenüber dieser Strategie bzw. die Notwendigkeit, sie durch andere - stärker dialogorientierte - Strategien zu ergänzen (Zimmermann 2013), weshalb es hier ebenfalls vonnöten ist, Misserfolge miteinzubeziehen, um mögliche (Kombinationen von) Erfolgsbedingungen, die (quasi-)hinreichend wirken, zu identifizieren.

Auch Arbeiten zur Überzeugung - einem Konzept, das auf der Einsichtsfähigkeit der Akteure beruht und Verhaltensänderungen auf echte Präferenzänderungen zurückführt (Checkel 2001: 562; Payne 2001: 42) - erforschen bislang ausschließlich Erfolgsfälle. Inspiriert von Habermas' Theorie verständigungsorientierten Handelns konzipiert Nicole Deitelhoff (2006: 73) Normunternehmer als situationsorientierte „Diskursmakler“, die darauf hinarbeiten, ,die normativen und institutionellen Bedingungen von Normgenerierungsprozessen so zu gestalten, dass sie den Anforderungen an rationale Diskurse nahe kommen" und der Sieg des besseren Arguments möglich wird (siehe auch Risse 1999: 534). Einige Autoren kritisieren, dass dieser Ansatz für das bessere Argument kein anderes Kriterium als das Ergebnis liefert (Hawkins 2004: 784; einschlägig Hanrieder 2008). Ebendiese Kriterien ex ante zu spezifizieren würde erlauben, solche Fälle zu erkennen, in denen sich bessere 
Argumente - trotz förderlicher Diskursbedingungen - nicht durchsetzen und nach den Ursachen ihres Scheiterns zu suchen. Außerdem bleibt nach wie vor offen, weshalb die Akteure unter gleichen Bedingungen dennoch unterschiedlich empfänglich für Überzeugungsversuche sind (Grobe 2010: 8).

\section{Die Wirkung von Normen: Effekte, Handlungslogiken und Mechanismen}

Die Wirksamkeit von Normen zeigt sich auf dreifache Weise: regulativ in der Einhaltung von Normen, konstitutiv in der Schaffung von Kategorien und Identitäten und strukturell in der Produktion eines normativen Fundaments der internationalen Politik (Wiener 2004: 189). Der folgende Abschnitt stellt die Erklärungen dieser Wirksamkeit vor und einige damit verbundene Probleme heraus.

\section{a) Regulative, konstitutive und permissive Effekte}

Während weitgehend Konsens darüber besteht, dass sich regulative, das heißt auf das Verhalten des Akteurs zielende, und konstitutive, das heißt bedeutungsstiftende, Wirkungen von Normen unterscheiden lassen, überraschen die damit einhergehenden Kausalitätsverständnisse mitunter. Zwar leuchtet der häufige Hinweis ein, dass Normen nicht zwangsläufig bestimmte Verhaltensweisen hervorbringen, sondern die Handlungsspielräume beschränken oder erweitern. ${ }^{9}$ Dass jedoch einige zentrale Autoren Normen deshalb jegliche Ursächlichkeit absprechen (z. B. Kratochwil/ Ruggie 1986: 767), muss als Fehlschluss aus einem offensichtlich engen, deterministischen Kausalitätsverständnis heraus kritisiert werden (Kurki 2006). So ist etwa die Konzeption von Normen als ermöglichende Bedingungen (Finnemore 1996: 158) nicht nachvollziehbar, weil diejenigen Handlungen, die durch Gebote und Verbote reguliert werden, auch schon vor der Existenz entsprechender Normen möglich sind. Ebensowenig überzeugt die Erklärung, Normen wirkten nicht kausal, weil sie zwar die Handlungsspielräume des Akteurs einschränkten, diesem aber immer noch eine Reihe verschiedener Handlungsoptionen ließen (Klotz 1995: 461 f.), denn offensichtlich liegt hier eine probabilistische Kausalität vor: Verbotsnormen senken die Wahrscheinlichkeit bestimmter Handlungen, während Gebotsnormen diese Wahrscheinlichkeit erhöhen (Kratochwil 1991: 100).

Freilich erschöpft sich in dieser kausalen Beziehung nicht das Wirkungsspektrum von Normen. So wirken sie auch konstitutiv bzw. bedeutungsschaffend - für Handlungen, Objekte und Akteure bzw. Akteursidentitäten (Searle 1995: 43 ff.; Ruggie 1998: 22). Demnach entstehen Identitäten bei der Normgenese (aber auch bei der

9 Kratochwil/Ruggie 1986: 767; Klotz 1995: 461 f;; Finnemore 1996: 158; Onuf 1997: 9. 
Normeinhaltung), wenn grundlegende gemeinsame Überzeugungen darüber herausgebildet werden, wer die Akteure sind und auf Basis welcher Werte sie agieren (Deitelhoff 2006: 45; Gurowitz 2006: 310). Daher sind Normen nicht regulativ oder konstitutiv, wie einige Autoren annehmen (Raymond 1997: 214; Finnemore/Sikkink 1998: 891; Alderson 2001: 421), sondern sie wirken immer regulativ und konstitutiv zugleich (Onuf 1998: 68).

Eine weitere - bislang unerforschte - Kategorie von Normeffekten sind sogenannte permissive Effekte. Ihre regulative Dimension bezeichnet die Fähigkeit mancher Normen, die internationale Aufmerksamkeit in einem solchen Maß zu beanspruchen, dass weitere Normen nicht beachtet werden. Ihre konstitutive Dimension liegt in der zwangsläufigen Legitimierung bestimmter Verhaltensweisen durch die Stigmatisierung anderer (Tannenwald 1999: 437; dies. 2007: 47). Nur wenige Arbeiten haben bislang das Erkenntnispotenzial genutzt, welches die Beachtung dieser nicht-intendierten Effekte von Normen birgt - sie können beispielsweise die Nicht-Entstehung oder die inkonsistente Anwendung anderer Normen erklären (Carpenter 2006).

\section{b) Konsequenzialitäts- und Angemessenheitslogik}

An die Frage nach der Wirkung von Normen schließt sich die Frage nach den Motiven der Akteure für deren Einhaltung an. Diese werden idealtypisch durch die Konsequenzialitätslogik (logic of consequences) und die Angemessenheitslogik (logic of appropriateness) erfasst (Deitelhoff 2006: 112). Die dahinterstehenden handlungstheoretischen Idealtypen, der homo oeconomicus und der homo sociologicus, wurden zwar in der Soziologie als Schreckensmänner (Weise 1989) und auch in den IB als reduktionistisch kritisiert (Herborth 2004: 62), sie stellen jedoch nach wie vor wichtige Referenzpunkte für die Normenforschung dar. Im Kern geht es dabei um die Frage, ob die Akteure danach streben, das für sich Beste oder das intersubjektiv Richtige zu tun. Die Handlungsmotive zu verstehen ist deshalb wichtig, weil davon die Entscheidung abhängt, welche Strategien man einsetzt, wenn man eine Änderung des Akteursverhaltens wünscht.

Nach der Rational-Choice-Theorie entscheiden sich Akteure auf Basis individueller Kosten-Nutzen-Kalküle und feststehender Präferenzen zwischen verschiedenen Handlungsalternativen für diejenige, die ihnen den größten Nutzen verspricht - sie orientieren sich damit an den antizipierten Konsequenzen einer Handlung für die eigenen und kollektiven Ziele (Elster 1989: 99; March/Olsen 1998: 949 f.). ${ }^{10}$

10 Dieses Akteurskonzept floss systematisch in die Regimetheorie ein; für eine Zusammenfassung der zentralen Annahmen siehe Hasenclever/Mayer/Rittberger 1997 sowie Müller 2013. 
Dabei kann die Präferenzordnung des Akteurs auch nicht-materielle Faktoren wie soziale Anerkennung, Status und Reputation enthalten (Akerlof 1980: 753 ff.; Bernheim 1994). Dem Einwand, die Integration des Sozialen widerspreche der individualistischen Ontologie, kann man entgegnen, dass nicht-materielle Gewinne letztendlich dem materiellen Nutzen dienten - wenn Kooperation zu Gewinnen führt, ist es schließlich im Interesse des rationalen Akteurs, seinen Ruf als verlässlicher Partner aufrechtzuerhalten, um nicht Gefahr zu laufen, von zukünftigen Kooperationen ausgeschlossen zu werden (McElroy 1992: 50). Ob mit schlechten „Compliern" tatsächlich weniger kooperiert wird, bleibt indes eine spannende Frage (Downs/Trento 2004: 24).

Ist im rationalistischen Menschenbild die Gesellschaft nicht mehr als ein Faktor auf der Nutzenfunktion des Akteurs, so ist sie in der durkheimschen Konzeption der sozialen Akteure „eine Macht, die sie bestimmt“ (Durkheim 1983: 271). Der Angemessenheitslogik folgende Akteure überlegen, in welcher Situation sie sich befinden, wer sie sind und welches Handeln für diese Situation angemessen ist (Sending 2002: 447), wobei die Angemessenheitsvorstellungen in Normen festgehalten, sozial geformt und durch Sozialisation vermittelt werden (March/Olsen 1989: 23). Es ist deshalb schwierig, die Angemessenheitslogik als Handlungsmotiv nachzuweisen, da selbst wenn Normen eingehalten werden, das Motiv ein anderes als die Norm selbst sein kann, etwa Unterwerfung oder Zwang oder schlichtes Eigeninteresse (Goertz/Diehl 1992: 635 ff.). Eine Möglichkeit, der Angemessenheitslogik auf die Spur zu kommen, liegt in der Auswahl solcher Fälle, in denen ein Akteur sein Verhalten nach der Entstehung einer Norm bei gleichbleibenden Interessen und Machtverhältnissen ändert oder eine andere Handlungsoption als die durch das Eigeninteresse naheliegende wählt, das heißt, auch unangenehme Pflichten beachtet (Oberthür 1996: 12). ${ }^{11}$ Eine weitere Möglichkeit ergibt sich durch die methodologische Verschiebung von Korrelationen und Kovarianzen hin zu Kausalmechanismen (Yee 1996: 85): Auch ohne alternative Erklärungen vorab eliminieren zu müssen, legt man dabei den Pfad offen, wie Normen ein bestimmtes Verhalten erzeugen (Checkel 2001).

Der Umgang der Normenforschung mit diesen Konzepten war von Beginn an integrativ in dem Sinne, dass die konsequenzialistische Logik durch die Angemessenheitslogik nicht grundsätzlich widerlegt, sondern ergänzt werden sollte. Erkennt man auch weiterhin an, dass beide Motive für unterschiedliche Akteure in unter-

11 Zahlreiche Artikel (z. B. Price 1998; Tannenwald 1999) wenden ebendiese Argumentationsstrategie an, um sich gegen rationalistische Einwände zu verteidigen, indem sie mit empirischen Puzzles einsteigen, welche mittels Interessen und Kosten-Nutzen-Kalkülen nicht zu erklären seien. 
schiedlichen Situationen in unterschiedlichem Maße handlungsleitend sein können, folgen weitere Forschungsfragen: Wie gehen die Akteure mit inneren Konflikten um, wenn die soziale Erwartung eine andere Handlungsoption nahelegt als das Eigeninteresse (Shannon 2000)? Was begünstigt dabei die Entscheidung für angemessenes und was für eigeninteressiertes Verhalten? Gibt es bestimmte Akteure, die sich häufiger als andere für bestimmte Optionen entscheiden und weshalb?

\section{c) Normenwirkung im internationalen System: Sozialisation, Lernen und Sanktionen}

Die Überlegung, über welche unterschiedlichen Logiken Normen auf das Akteursverhalten Einfluss nehmen können, führt zu der Frage, über welche Mechanismen sich die Akteure der Existenz der im internationalen System vorhandenen Normen bewusst werden und welche Faktoren die Verhaltensanpassung begünstigen oder gar erzwingen.

Zwei prominente Erklärungsmechanismen sind Sozialisation und Lernen, wobei Sozialisation den gesamten Prozess, Lernen die Vorgänge im Akteur meint. In Anlehnung an die soziologische Sozialisationstheorie wird darunter die Vergesellschaftung der Akteure verstanden: Sie werden durch Interaktionsprozesse an das soziale Leben mit seinen Werten, Normen und Rollen herangeführt. Noch vor der Normenforschung hatte bereits die englische Schule das internationale System als Gesellschaft konzipiert (Bull 1977), die sich durch gemeinsame Interessen und Zielvorstellungen auszeichnet und über Regeln, die der Erreichung dieser Ziele dienen, sowie über Institutionen der Regelumsetzung verfügt (McElroy 1992: 35). Das internationale System wird zur Sozialisationsinstanz, die über den Zugang zu sozialen Ressourcen entscheidet; einzelne Akteure zu Sozialisanden, die (nicht unbedingt bewusst) nach diesem Zugang streben und sich deshalb den Überzeugungen und Erwartungen anpassen (Schimmelfennig 2000: 117). Sozialisation ist demnach ein Lernprozess, in dem Ideen in Interaktionen zwischen lernfähigen Parteien durch Imitation der Mehrheit oder der Vorbilder (Bikchandani/Hirshleifer/Welch 1992: 994; Bernheim 1994: 842; Rost Rublee 2008: 423) - übertragen und internalisiert werden (Finnemore 1993; Checkel 1998: 4; Schimmelfennig 2000: $111 \mathrm{f}$.).

Sozialisation und Lernen erscheinen als plausible Mechanismen, mit denen man vor allem Erfolgsfälle erklären kann - der Verfeinerungsbedarf ergibt sich hier jedoch wiederholt daraus, dass sie recht wenig Aufschluss über abweichende Fälle geben: Warum wirken internationale Sozialisationsprozesse nicht auf alle Akteure gleichermaßen und stoßen überall Lernprozesse an? Schließlich gibt es einerseits Akteure, die trotz hoher Interaktionsdichte gegenüber internationalen Normen re- 
lativ resistent bleiben und andererseits Akteure, die sich auch ohne häufigen Kontakt zu Sozialisationsinstanzen weitgehend an den Normenkatalog halten.

Auch Modelle, die diese Mechanismen in Erklärungen von Diffusionsprozessen von Normen integrieren, bedürfen weiterer Spezifikation. So erfreut sich das NormLife-Cycle (Finnemore/Sikkink 1998) häufiger Anwendung (etwa Ingebritsen 2002; Björkdahl 2002; Kelley 2008), jedoch hat bisher niemand überprüft, inwiefern die Normkaskade bzw. der tipping point, nach dessen Erreichen die Zahl der Normunterstützer exponentiell wächst, tatsächlich charakteristisch für Diffusionsprozesse ist. Die Autorinnen führen die Einführung des Frauenwahlrechts und die Unterstützung der Landminenkonvention zwar als Beispiele an, bei anderen Prozessen jedoch vermisst man diesen Punkt. ${ }^{12}$ Das widerlegt zwar nicht die Möglichkeit einer Kaskade, demonstriert jedoch, dass auch theoretisch griffige Konzepte nach einem über Einzelfälle hinausgehenden empirischen Nachweis verlangen und die Identifikation der Bedingungen für eine solche Kaskade notwendig ist. Auch mit Blick auf die dritte Phase - die Internalisierung - verliert das Modell an Stringenz: Hier wechseln die Autorinnen von der internationalen zur nationalen Ebene, von der quantitativen Anerkennung der Norm zur qualitativen und von der normzentrierten zu einer akteurszentrierten Perspektive. Dadurch lassen sich Phase 2 und 3 nicht mehr als distinkte Phasen unterscheiden, denn während die Norm noch international diffundiert, kann sie von einzelnen Akteuren bereits internalisiert werden. Die passende dritte Stufe wäre daher das Ende der Kaskadenbewegung, das heißt die weitgehend universelle Anerkennung (und nicht die individuelle Internalisierung) der Norm. Einige Arbeiten haben außerdem aufgezeigt, dass Akteure die Macht haben, die Gültigkeit internalisierter Normen im Diskurs zu hinterfragen und damit deren Erosion auszulösen, wodurch bezweifelt werden darf, dass Internalisierung tatsächlich den Endpunkt einer Normkarriere darstellt (Rosert/Schirmbeck 2007; McKeown 2009).

Während das Norm-Life-Cycle mögliche Diffusionshindernisse ausblendet und den Sozialisationsprozess wie einen Selbstläufer erscheinen lässt, befassen sich Studien zur Normdurchsetzung (norm enforcement) mit „compliance gaps“, die entstehen, weil manche Akteure nicht von der Kaskadendynamik erfasst werden (Downs/Trento 2004: 19). Als Durchsetzung zählen Tätigkeiten zur Verringerung der Diskrepanz zwischen Norm und Verhalten, sodass schließlich eine „nicht gänzlich freiwillige Normbeachtung erreicht wird“ (Zangl 2001: 52). Dies geschieht

12 Beispielsweise gab es bei der Chemiewaffenkonvention einen mehr oder weniger gleichmäßigen Anstieg der Ratifikationen. 
mittels verschiedener Sanktionsmechanismen, ${ }^{13}$ deren trans-, inter- und nationales Zusammenspiel im „Spiralmodell des Menschenrechtswandels“ erfasst wird (Forschungsgruppe Menschenrechte 1998; Risse/Ropp/Sikkink 1999). Im Kern setzt sanktionsbasierte Sozialisation offenkundig auf einen rationalen Akteur, dessen Kosten-Nutzen-Kalküle durch Drohungen verändert werden (Zangl 2001: 53). Als weicher Mechanismus gelten soziale Sanktionen (Schimmelfennig 2001: 64) wie der Abbruch diplomatischer Kontakte und die Kritik am Fehlverhalten. Als harter Mechanismus gelten materielle Sanktionen wie finanzielle, wirtschaftliche oder militärische Zwangsmaßnahmen. Da Versuche, die Erfolgsaussichten sanktionsbasierter Normendurchsetzung nach Akteurstypen zu differenzieren (De Nevers 2007), vergleichsweise neu sind, besteht hier Forschungsbedarf. Auch unterschiedliche Gründe für Normbrüche und subtilere Formen der Compliance-Förderung sollten mehr beachtet werden: Bei Unwilligkeit mag Zwang die aussichtsreichste Option sein, bei Unfähigkeit ist es jedoch Unterstützung (Franck 1990; Chayes/ Handler Chayes 1993: 24; Zangl 2001: 54).

\section{d) Normenwirkung im Staat und in Regionen: Nationale Eliten und Lokali- sierung}

Eine zweifache Varianz - die ungleiche Akzeptanz gleicher Normen im inter- und im intraregionalen Vergleich und die ungleiche Akzeptanz unterschiedlicher Normen im gleichen nationalen Kontext (Acharya 2004: 240; McIntosh Sundstrom 2005: 419) - hat als Ansporn gedient, Sozialisation als komplexen Prozess zu begreifen, der auf verschiedenen Ebenen abläuft: neben der internationalen auch auf Ebene der nationalen Eliten und der nationalen Bevölkerung bis hin zu einzelnen Individuen (Flockhart 2006: 93, 99). Anders als das Spiralmodell steigen diese Ansätze nicht erst bei Compliance-Problemen ein, sondern schon beim Erscheinen der Norm auf der innenpolitischen Tagesordnung, womit das Mitwirken nationaler Akteure - oder, klassisch liberal, unterschiedlicher Interessensgruppen - bei der Formierung normativer Vorstellungen im Rahmen ihrer institutionellen Handlungsmöglichkeiten ins Zentrum rückt (Cortell/Davis Jr. 1996: 453; Checkel 1997: 476). Durch die Untersuchung des politischen Systems kann erklärt werden, wie Normen diffundieren, warum Diffusionsprozesse ausbleiben und warum es in manchen Systemen eher eine top-down-Diffusion, in anderen hingegen eine bottom-up-Diffusion gibt (Checkel 2001: 580).

$13 \mathrm{Zu}$ Sanktionen gibt es in den Internationalen Beziehungen eine breite Debatte, die vor die Anfänge der Normenforschung zurückreicht bzw. parallel geführt wird (etwa Baldwin 1971; Cortright/ Lopez 1995). 
Es bleibt allerdings nach wie vor offen, weshalb einige Normen innenpolitisch verfangen und andere nicht. Als Erklärungsfaktor hierfür wird die lokale Passung von internationalen Normen identifiziert: Können sie an bestehende innerstaatliche Vorstellungen andocken, kann die Diffusion inkrementell vonstattengehen; widersprechen sie ihnen hingegen, sind vermehrte Mobilisierungsanstrengungen und Druck notwendig (Farrell 2001: 65, 81) oder die Diffusion misslingt gänzlich (McIntosh Sundstrom 2005: $432 \mathrm{f}$.). Während in früheren Arbeiten Passung bzw. Nicht-Passung als entweder vorhanden oder nicht vorhanden erscheinen (Lutz/Sikkink 2000: 659), stößt neuerdings die aktive Herstellung der Passung durch die innenpolitischen Akteure auf Interesse. Die stabile, strukturierende und zugleich umstrittene Bedeutung von Normen ermöglicht kontextabhängige Interpretationen (Wiener 2004, 2007) und damit Lokalisierungsprozesse - die diskursive Integration fremder Normen in lokale Kontexte mit dem Ergebnis der Übereinstimmung beider (Acharya 2004: 245, 269). Gemeint ist damit keine Einbahnstraße im Sinne einer mit lokalen Normen kompatiblen Auslegung der internationalen Norm, sondern eine wechselseitige Anpassung (Hui-Jung Kim 2009: 679). Auf diese Weise legitimieren Normen solche Handlungen, für deren Rechtfertigung das lokale normative Repertoire allein nicht ausreicht (Gurowitz 2006: 305); zugleich werden normative Auseinandersetzungen bzw. Normkonflikte zum Erklärungsfaktor für Normenwandel (Sandholtz/Stiles 2009; Müller/Wunderlich 2013). Während der Fokus auf lokale Prozesse der Normenwirkung und -transformation auch weiterhin fruchtbar zu bleiben verspricht, wie die Vielzahl dadurch inspirierter Forschungsarbeiten zeigt, ${ }^{14}$ gilt es erstens, im Auge zu behalten, dass „Lokalisierung“ und „kulturelle Übersetzung“ von Normen zum Feigenblatt für solche Interpretationen von Normen werden können, die ihren normativen Gehalt schwächen, wie einige Studien (Capie 2008) belegen. Zweitens wurde Umstrittenheit bisher vor allem auf lokaler bzw. regionaler Ebene untersucht und entsprechend die Unterschiede im globalen Entstehungskontext und im lokalen Auslegungs- bzw. Umsetzungskontext als Quellen der Umstrittenheit identifiziert. Erst wenige Arbeiten untersuchen Umstrittenheit auf globaler Ebene bzw. im gleichen organisationalen Kontext (Contessi 2010; Krook/True 2010); ebenso steht ein Vergleich der lokalen und globalen Dynamiken und Quellen von Umstrittenheit noch aus. 


\section{Bilanz: Würdigung und Forschungsdesiderate}

Die Forschung über Normen in den Internationalen Beziehungen hat viele Verdienste. Sie hat (zusammen mit der Regimetheorie und der Global-GovernanceForschung) den Blickwinkel auf die internationale Politik mehrfach erweitert: Zwar war es bereits der Regimeforschung gelungen, mit Umweltpolitik ein „softes“ Politikfeld in den IB zu etablieren, doch blieb hier aufgrund des grenzüberschreitenden Charakters vieler Umweltprobleme die zwischenstaatliche Dimension im Fokus. Durch die Normenforschung fand mit den Menschenrechten ein weiterer ,softer“ Bereich Eingang in die Internationalen Beziehungen - und zudem einer, in dessen Zentrum nicht die Lösung von Problemen kollektiven Handelns steht.

Neben dem empirischen brachte die Normenforschung auch einen erheblichen theoretischen Mehrwert für die IB. Die Prävalenz und Relevanz sozialer Verhaltensmuster zwischen Staaten wurde aufgezeigt und deren Handlungsmotivationen wurden ausdifferenziert, indem moralische Beweggründe und das Bestreben, sich sozial angemessen zu verhalten und das Richtige zu tun, auch für sie geltend gemacht wurden. Durch die Relativierung des skeptischen Bildes von Staaten als egoistisch und, was die eigene Sicherheit betrifft, paranoid wurde es möglich, Fortschrittsperspektiven für die internationale Politik zu eröffnen wie auch tatsächliche Fortschritte zu erklären.

An Ansatzpunkten für weitere Forschung mangelt es immer noch nicht, weil der für die Normenforschung charakteristische Fortschrittsglaube (Brabandt 2011: 43) im Zusammenspiel mit der dezidierten Abgrenzung von bisherigen Paradigmen zur Entstehung einiger empirischer und methodologischer blinder Flecken geführt hat. So hat der ,nice norms bias“ (Finnemore/Sikkink 2001: 404; McKeown 2009: 7) den Blick für die Schwächung bereits etablierter Normen (Normerosion), solche Effekte von Normen, die von ihren Proponenten nicht intendiert waren (permissive Effekte), wie auch für gescheiterte Prozesse der Normentstehung oder die Problematik der Normen- und Netzwerkkonkurrenz verstellt. Ungleichgewichte gibt es auch bei der Wahl der Politikfelder: Studien aus den Bereichen Sicherheit und Menschenrechte sind reichlich vorhanden, im Gegensatz zu den wenigen Studien über Normen aus den Bereichen Wirtschaft (Yanacopulos 2004) oder Umwelt (Bailey 2008). Für Akteure findet man einseitige Rollenzuschreibungen: So werden Staaten vor allem als Normadressaten, Internationale Organisationen vor allem als Normlehrer und Nicht-Regierungsorganisationen vor allem als Normunternehmer betrachtet. In methodologischer Hinsicht war zwar die Vorliebe für Einzel- und small-n-Fallstudien - sogenannte ,small-t truth claims“ (Finnemore/Sikkink 2001: 394) - ausgesprochen wichtig für die Heuristik, aber angesichts der inzwischen 
erreichten Stabilität des theoretischen Gerüstes wäre weniger Generalisierungsvorsicht sinnvoll, um die identifizierten Kausalmechanismen und die notwendigen und hinreichenden Bedingungen bestimmter Prozesse und Effekte anhand größerer Vergleichsstudien zu systematisieren. Ferner liegt es nahe, die beiden großen Forschungsschwerpunkte - die Entstehung von Normen und deren Wirkung - stärker miteinander zu verbinden und Zusammenhänge zwischen Normtypen, Entstehungsarten und Compliance-Graden zu untersuchen.

Kurzum: Unser Bild von der internationalen Welt, den dort relevanten Problemen, den dort agierenden Akteuren und zutage tretenden Wirkungsmechanismen wurde durch die Normenforschung vollständiger. Doch auch für diesen Forschungsstrang gilt das, was für die Wissenschaft im Allgemeinen gilt: Die Beantwortung von Fragen wirft - erfreulicherweise - immer auch neue Fragen auf.

\section{Literatur}

Acharya, Amitav, 2004: How Ideas Spread: Whose Norms Matter? Norm Localization and Institutional Change in Asian Regionalism, in: International Organization 58 (2), 239-275.

Akerlof, George, 1980: A Theory of Social Custom, of Which Unemployment May

Be One Consequence, in: Quarterly Journal of Economics 94 (4), 749-775.

Alderson, Kai, 2001: Making sense of state socialization, in: Review of International Studies 27 (3), 415-433.

Axelrod, Robert, 1986: An Evolutionary Approach to Norms, in: American Political

Science Review 80 (4), 1095-1111.

Bailey, Jennifer L., 2008: Arrested Development: The Fight to End Commercial

Whaling as a Case of Failed Norm Change, in: European Journal of International

Relations 14 (2), 289-318.

Baldwin, David, 1971: The Power of Positive Sanctions, in: World Politics 24 (1), 19-38.

Bearce, David H./Bondanella, Stacy, 2007: Intergovernmental Organizations, Socialization, and Member-State Interest Convergence, in: International Organization 61 (4), 703-733.

Bernheim, Douglas B., 1994: A Theory of Conformity, in: The Journal of Political Economy 102 (5), 841-877.

Bikchandani, Sushil/Hirshleifer, David/Welch, Ivo, 1992: A Theory of Fads, Fashion, Custom, and Cultural Change as Informational Cascades, in: The Journal of Political Economy 100 (5), 992-1026.

Björkdahl, Annika, 2002: From Idea to Norm. Promoting Conflict Prevention, Lund. 
Brabandt, Heike, 2011: Internationale Normen und das Rechtssystem: der Umgang mit geschlechtsspezifisch Verfolgten in Großbritannien und Deutschland, Baden-Baden.

Bull, Hedley, 1977: The Anarchical Society: A Study of Order in World Politics, London/Basingstoke.

Capie, David, 2008: Localization as Resistance: The Contested Diffusion of Small Arms Norms in Southeast Asia, in: Security Dialogue 39 (6), 637-658.

Carpenter, R. Charli, 2006: Innocent Women and Children: Gender, Norms and the Protection of Civilians, Aldershot.

Carpenter, R. Charli, 2007 a: Setting the Advocacy Agenda: Theorizing Issue Emergence and Nonemergence in Transnational Advocacy Networks, in: International Studies Quarterly 51 (1), 99-120.

Carpenter, R. Charli, 2007 b: Studying Issue (Non)-Adoption in Transnational Advocacy Networks, in: International Organization 61 (3), 643-667.

Carpenter, R. Charli, 2011: Vetting the Advocacy Agenda: Network Centrality and the Paradox of Weapons Norms, in: International Organization 65 (1), 69-102.

Chayes, Abram/Handler Chayes, Antonia, 1993: On Compliance, in: International Organization 47 (2), 175-205.

Checkel, Jeffrey T., 1997: International Norms and Domestic Politics: Bridging the Rationalist-Constructivist Divide, in: European Journal of International Relations 3 (4), 473-495.

Checkel, Jeffrey T., 1998: International Norms and Domestic Agents: Probing the Dynamics of Socialization. Paper Presented at the Ideas, Culture and Political Analysis Workshop, Princeton University, 15.-16.5.1998, http:// www.ciaonet.org/conf/ssr01/ssr01al.html (Stand: 16.4.2013).

Checkel, Jeffrey T., 1999: Norms, Institutions, and National Identity in Contemporary Europe, in: International Studies Quarterly 43 (1), 83-114.

Checkel, Jeffrey T., 2001: Why Comply? Social Learning and European Identity Change, in: International Organization 55 (3), 553-588.

Cho, Eunjeong, 2012: EURATOM: nuclear norm competition between allies, 1955-1957, unpublished PhD Thesis.

Reus-Smit, Christian/Snidal, Duncan, 2008: Between Utopia and Reality: The Practical Discourses of International Relations, in: Christian Reus-Smit/Duncan Snidal (Hrsg.), The Oxford handbook of international relations, Oxford, 3-37.

Clark, Ann Marie, 2001: Diplomacy of Conscience: Amnesty International and Changing Human Rights Norms, Princeton. 
Contessi, Nicola P., 2010: Multilateralism, Intervention and Norm Contestation: China's Stance on Darfur in the UN Security Council, in: Security Dialogue 41 (3), 323-344.

Cooley, Alexander/Ron, James, 2002: The NGO Scramble - Organizational Insecurity and the Political Economy of Transnational Action, in: International Security 27 (1), 5-39.

Cortell, Andrew P./Davis Jr., James W., 1996: How Do International Institutions Matter? The Domestic Impact of International Rules and Norms, in: International Studies Quarterly 40 (4), 451-478.

Cortright, David/Lopez, George A., 1995: Economic Sanctions: Panacea or Peacebuilding in a Post-Cold War World, Boulder.

De Nevers, Renee, 2007: Imposing International Norms: Great Powers and Norm Enforcement, in: International Studies Review 9 (1), 53-80.

Deitelhoff, Nicole, 2006: Überzeugung in der Politik: Grundzüge einer Diskurstheorie internationalen Regierens, Frankfurt am Main.

Deitelhoff, Nicole, 2009: The Discursive Process of Legalization: Charting Islands of Persuasion in the ICC Case, in: International Organization 63 (1), 33-65.

Donno, Daniela, 2010: Who is Punished? Regional Intergovernmental Organizations and the Enforcement of Democratic Norms, in: International Organization 64 (4), 593-625.

Downs, George W./Trento, Andrea W., 2004: Conceptual Issues Surrounding the Compliance Gap, in: Edward C. Luck/Michael W. Doyle (Hrsg.), International Law and Organization: Closing the Compliance Gap, Lanham, 19-40.

Durkheim, Emile, 1983: Der Selbstmord, Frankfurt am Main.

Elgström, Ole, 2009: Trade and aid? The negotiated construction of EU policy on economic partnership agreements, in: International Politics 46 (4), 451-468. Elster, Jon, 1989: The Cement of Society. A Study of Social Order, Cambridge.

Farrell, Theo, 2001: Transnational Norms and Military Development: Constructing Ireland's Professional Army, in: European Journal of International Relations 7 (1), 63-102.

Finnemore, Martha, 1993: International organizations as teachers of norms: the United Nations Educational, Scientific, and Cultural Organization and science policy, in: International Organization 47 (4), 565-598.

Finnemore, Martha, 1996: Constructing Norms of Humanitarian Intervention, in:

Peter J. Katzenstein (Hrsg.), The Culture of National Security: Norms and Identity in World Politics, New York, 153-185.

Finnemore, Martha/Sikkink, Kathryn, 1998: International Norm Dynamics and Political Change, in: International Organization 52 (4), 887-917. 
Finnemore, Martha/Sikkink, Kathryn, 2001: Taking Stock: The Constructivist Research Program in International Relations and Comparative Politics, in: Annual Review of Political Science 4 (1), 391-416.

Flockhart, Trine, 2006: 'Complex Socialization': A Framework for the Study of State Socialization, in: European Journal of International Relations 12 (1), 89-118.

Flohr, Annegret/Rieth, Lothar/Schwindenhammer, Sandra/Wolf, Klaus Dieter, 2010: The Role of Business in Global Governance. Corporations as Norm-Entrepreneurs, Basingstoke/Houndmills.

Florini, Ann, 1996: The Evolution of International Norms, in: International Studies Quarterly 40 (3), 363-389.

Forschungsgruppe Menschenrechte, 1998: Internationale Menschenrechtsnormen, transnationale Netzwerke und politischer Wandel in den Ländern des Südens, in: Zeitschrift für Internationale Beziehungen 5 (1), 5-41.

Franck, Thomas M., 1990: The Power of Legitimacy Among Nations, New York.

Goertz, Gary/Diehl, Paul F., 1992: Toward a Theory of International Norms: Some Conceptual and Measurement Issues, in: Journal of Conflict Resolution 36 (4), 634-664.

Greenhill, Brian, 2010: The Company You Keep: International Socialization and the Diffusion of Human Rights Norms, in: International Studies Quarterly 54 (1), 127-145.

Grobe, Christian, 2010: The power of words: Argumentative persuasion in international negotiations, in: European Journal of International Relations 16 (1), 5-29.

Gurowitz, Amy, 2006: The Diffusion of International Norms: Why Identity Matters, in: International Politics 43 (3), 305-341.

Haas, Peter M., 1989: Do Regimes Matter? Epistemic Communities and Mediterranean Pollution Control, in: International Organization 43 (3), 377-403.

Hafner-Burton, Emilie M., 2008: Sticks and Stones: Naming and Shaming the Human Rights Enforcement Problem, in: International Organization 62 (4), 689-716.

Hanrieder, Tine, 2008: Moralische Argumente in den Internationalen Beziehungen. Grenzen einer verständigungstheoretischen 'Erklärung' moralischer Debatten, in: Zeitschrift für Internationale Beziehungen 15 (2), 161-186.

Hasenclever, Andreas/Mayer, Peter/Rittberger, Volker, 1997: Theories of International Regimes, Cambridge. 
Hawkins, Darren, 2004: Explaining Costly International Institutions: Persuasion and Enforceable Human Rights Norms, in: International Studies Quarterly 48 (4), 779-804.

Herborth, Benjamin, 2004: Die via media als konstitutionstheoretische Einbahnstraße. Zur Entwicklung des Akteur-Struktur-Problems bei Alexander Wendt, in: Zeitschrift für Internationale Beziehungen 11 (2), 61-87.

Herr, Stefanie, 2010: Vom Regelbruch zu politischer Verantwortung. Die Anerkennung völkerrechtlicher Normen durch nicht-staatliche Gewaltakteure im Sudan, HSFK-Report 5/2010, Frankfurt am Main.

Hofferberth, Matthias/Brühl, Tanja/Burkart, Erik/Fey, Marco/Peltner, Anne, 2011: Multinational Enterprises as "Social Actors" - Constructivist Explanations for Corporate Social Responsibility, in: Global Society 25 (2), 205-226.

Hui-Jung Kim, Nora, 2009: Framing multiple others and international norms: the migrant worker advocacy movement and Korean national identity reconstruction, in: Nations and Nationalism 15 (4), 678-695.

Hurrell, Andrew/Macdonald, Terry, 2013: Ethics and Norms in International Relations, in: Walter Carlsnaes/Thomas Risse/Beth Simmons (Hrsg.), Handbook of International Relations, London, 57-83.

Hyde, Susan, 2011: Catch Us If You Can: Election Monitoring and International Norm Diffusion, in: American Journal of Political Science 55 (2), 356-369.

Ingebritsen, Christine, 2002: Norm Entrepreneurs: Scandinavia's Role in World Politics, in: Cooperation and Conflict 37 (1), 11-23.

Jepperson, Ronald R./Wendt, Alexander/Katzenstein, Peter, 1996: Norms, Identity and Culture in National Security, in: Peter J. Katzenstein (Hrsg.), The Culture of National Security: Norms and Identity in World Politics, New York, 33-75.

Kamis, Ben, 2011: Towards a Theory of Emergent International Conventions: Inducing from the Three-Mile Rule of Territorial Seas. Paper presented at the International Studies Association Annual Conference, Montreal, 16.-19.3.2011, $\mathrm{http}: / /$ citation.allacademic.com//meta/p_mla_apa_research_citation $/ 5 / 0 / 0 / 6 / 1 /$ pages500617/p500617-1.php (Stand: 16.4.2013).

Katzenstein, Peter, 1996: Cultural norms and national security: police and military in postwar Japan, Ithaca/London.

Kaufmann, Chaim D./Pape, Robert A., 1999: Explaining Costly International Moral Action: Britain's Sixty-year Campaign against the Atlantic Slave Trade, in: International Organization 53 (4), 631-668.

Keck, Margaret E.Sikkink, Kathryn, 1998: Activists Beyond Borders. Advocacy

Networks in International Politics, Ithaca. 
Kelley, Judith, 2008: Assessing the Complex Evolution of Norms: The Rise of International Election Monitoring, in: International Organization 62 (2), 221-255. Klotz, Audie, 1995: Norms Reconstituting Interests: Global Racial Equality and U.S. Sanctions against South Africa, in: International Organization 49 (3), 451-478. Klotz, Audie, 1996: Norms in International Relations. The Struggle Against Apartheid, Ithaca/London.

Krasner, Stephen, 1983: Structural Causes and Regime Consequences: Regimes as Intervening Variables, in: Stephen Krasner (Hrsg.), International Regimes, Ithaca/London, 1-21.

Kratochwil, Friedrich/Ruggie, John Gerard, 1986: International Organization: A State of the Art on an Art of the State, in: International Organization 40 (4), 753-775.

Kratochwil, Friedrich V., 1991: Rules, Norms, and Decisions. On the Conditions of Practical and Legal Reasoning in International Relations and Domestic Affairs, Cambridge.

Krook, Mona Lena/True, Jacqui, 2010: Rethinking the life cycles of international norms: The United Nations and the global promotion of gender equality, in: European Journal of International Relations 17 (2), 1-25.

Kurki, Milja, 2006: Causes of a Divided Discipline: Rethinking the Concept of Cause in International Relations Theory, in: Review of International Studies 32 (2), 189-216.

Legro, Jeffrey, 1997: Which Norms Matter: Revising the 'Failure' of Internationalism, in: International Organization 51 (1), 31-63.

Liese, Andrea, 2006: Staaten am Pranger: zur Wirkung internationaler Regime auf die innerstaatliche Menschenrechtspolitik, Wiesbaden.

Lutz, Ellen S./Sikkink, Kathryn, 2000: International Human Rights Law and Practice in Latin America, in: International Organization 54 (3), 633-659.

March, James G./Olsen, Johan P., 1989: Rediscovering Institutions, New York.

March, James G./Olsen, Johan P., 1998: The Institutional Dynamics of International Political Orders, in: International Organization 52 (4), 943-969.

McElroy, Robert W., 1992: Morality and American Foreign Policy: The Role of Ethics in International Affairs, Princeton.

McIntosh Sundstrom, Lisa, 2005: Foreign Assistance, International Norms, and NGO Development: Lessons from the Russian Campaign, in: International Organization 59 (2), 419-450.

McKeown, Ryder, 2009: Norm Regress: US Revisionism and the Slow Death of the Torture Norm, in: International Relations 23 (5), 5-25. 
Moore, Frances C., 2012: Negotiating Adaptation: Norm Selection and Hybridization in International Climate Negotiations, in: Global Environmental Politics 12 (4), 30-48.

Müller, Harald, 2013: Security Cooperation, in: Walter Carlsnaes/Thomas Risse/ Beth Simmons (Hrsg.), Handbook of International Relations, London, 607-635. Müller, Harald/Wunderlich, Carmen (Hrsg.), 2013: Norm Dynamics in Multilateral Arms Control. Interests, Conflicts, and Justice, Athens.

Nadelmann, Ethan, 1990: Global Prohibition Regimes: The Evolution of Norms in International Society, in: International Organization 44 (4), 479-526.

Oberthür, Sebastian, 1996: Die Reflexivität internationaler Regime. Erkenntnisse aus der Untersuchung von drei umweltpolitischen Problemfeldern, in: Zeitschrift für Internationale Beziehungen 3 (1), 7-44.

Onuf, Nicholas, 1997: A Constructivist Manifesto, in: Kurt Burch/Robert A. Denemark (Hrsg.), Constituting International Political Economy, London, 7-19.

Onuf, Nicholas, 1998: Constructivism: A user's manual, in: Vendulka Kubálková/ Nicholas Onuf/Paul Kowert (Hrsg.), International relations in a constructed world, Armonk, 58-78.

Payne, Rodger A., 2001: Persuasion, Frames and Norm Construction, in: European Journal of International Relations 7 (1), 37-61.

Price, Richard, 1998: Reversing the Gun Sights: Transnational Civil Society Targets Land Mines, in: International Organization 52 (3), 613-644.

Raymond, Gregory A., 1997: Problems and Prospects in the Study of International Norms, in: Mershon International Studies Review 41 (2), 205-245.

Risse, Thomas, 1999: International Norms and Domestic Change: Arguing and Communicative Behavior in the Human Rights Area, in: Politics \& Society 27 (4), 529-560.

Risse, Thomas/Ropp, Stephen/Sikkink, Kathryn (Hrsg.), 1999: The Power of Human Rights: International Norms and Domestic Change, Cambridge.

Risse, Thomas/Sikkink, Kathryn/Ropp, Steven (Hrsg.), 2013: The Persistent Power of Human Rights, Cambridge.

Rosert, Elvira, 2011: Norms matter - but not only in the way they are supposed to: exploring unintended effects of international norms, International Studies Association Convention (ISA), Montreal, 16.-19.3.2011, http:// www.gesellschaftswissenschaften.uni-frankfurt.de/uploads/images/1707/ rosert_isa2011.pdf (Stand: 16.4.2013).

Rosert, Elvira/Schirmbeck, Sonja, 2007: Zur Erosion internationaler Normen: Folterverbot und nukleares Tabu in der Diskussion, in: Zeitschrift für Internationale Beziehungen 14 (2), 253-287. 
Rost Rublee, Maria, 2008: Taking Stock of the Nuclear Nonproliferation Regime: Using Social Psychology to Understand Regime Effectiveness, in: International Studies Review 10 (3), 420-450.

Ruggie, John Gerard, 1998: Constructing the world polity: essays on international institutionalization, London/New York.

Rutherford, Kenneth R./Brem, Stefan/Matthew, Richard A. (Hrsg.), 2003: Reframing the Agenda: The Impact of NGO and Middle Power Cooperation in International Security Policy, Westport.

Sandholtz, Wayne, 2008: Dynamics of International Norm Change: Rules against Wartime Plunder, in: European Journal of International Relations 14 (2), 101-131.

Sandholtz, Wayne/Stiles, Kendall, 2009: International Norms and Cycles of Change, Oxford.

Santa-Cruz, Arturo, 2005: Constitutional Structures, Sovereignty, and the Emergence of Norms: The Case of International Election Monitoring, in: International Organization 59 (2), 663-693.

Schimmelfennig, Frank, 2000: International Socialization in the New Europe, in: European Journal of International Relations 6 (1), 109-139.

Schimmelfennig, Frank, 2001: The Community Trap: Liberal Norms, Rhetorical Action, and the Eastern Enlargement of the European Union, in: International Organization 55 (1), 47-80.

Searle, John R., 1995: The Construction of Social Reality, New York.

Sending, Ole Jacob, 2002: Constitution, Choice and Change: Problems with the 'Logic of Appropriateness' and its Use in Constructivist Theory, in: European Journal of International Relations 8 (4), 443-470.

Shannon, Vaughn P., 2000: Norms Are What States Make of Them: The Political Psychology of Norm Violation, in: International Studies Quarterly 44 (2), 293-316.

Sigman, Rachel/Schmitz, Hans Peter/Mergel, Ines, 2011: Networks versus Issues: The Evolution of the Campaign to Defeat Diarrheal Disease. International Studies Association Convention, Montreal, 16.-19.3.2011, http://citation.allacademic.com/meta/p_mla_apa_research_citation/5/0/0/5/0/pages500502/ p500502-1.php (Stand: 16.4.2013).

Sikkink, Kathryn, 2008: The role of consequences, comparison and counterfactuals in constructivist ethical thought, in: Richard Price (Hrsg.), Moral Limit and Possibility in World Politics, Cambridge, 83-111.

Tannenwald, Nina, 1999: The Nuclear Taboo: The United States and the Normative Basis of Nuclear Non-Use, in: International Organization 53 (3), 433-468. 
Tannenwald, Nina, 2007: The Nuclear Taboo: The United States and the Non-Use of Nuclear Weapons since 1945, Cambridge.

Tholens, Simone, 2013: Localization Strategies in Post-War Security Governance: Bringing in State-Society Narratives. Paper prepared for the ISA Annual Convention 2013, San Francisco, 3.-6.4.2013, http://files.isanet.org/ConferenceArchive/aa51ca6bdbe3429d9453d03daf57d7ba.pdf (Stand: 16.4.2013).

Thomson, Janice E., 1993: Norms in International Relations: A Conceptual Analysis, in: International Journal of Group Tensions 23 (1), 67-83.

Ullmann-Margalit, Edna, 1977: The Emergence of Norms, Oxford.

Weise, Peter, 1989: Homo oeconomicus und homo sociologicus. Die Schreckensmänner der Sozialwissenschaften, in: Zeitschrift für Soziologie 18 (2), 148-161.

Wiener, Antje, 2004: Contested Compliance: Interventions on the Normative Structure of World Politics, in: European Journal of International Relations 10 (2), 189-234.

Wiener, Antje, 2007: The Dual Quality of Norms and Governance beyond the State: Sociological and Normative Approaches to Interaction, in: Critical Review of International Social and Political Philosophy 10 (1), 47-69.

Wiener, Antje, 2009: Enacting meaning-in-use: qualitative research on norms and international relations, in: Review of International Studies 35 (1), 175-194.

Wohlforth, William C., 1994: Realism and the End of the Cold War, in: International Security 19 (3), 91-129.

Wunderlich, Carmen, 2013: Theoretical Approaches in Norm Dynamics, in: Harald Müller/Carmen Wunderlich (Hrsg.), Norm Dynamics in Multilateral Arms Control, Athens, 20-47.

Yanacopulos, Helen, 2004: The Public Face of Debt, in: Journal of International Development 16 (5), 717-727.

Yee, Albert S., 1996: The Causal Effects of Ideas on Policies, in: International Organization 50 (1), 69-108.

Zangl, Bernhard, 2001: Bringing Courts Back In: Normdurchsetzung im GATT, in der WTO und der EG, in: Swiss Political Science Review 7 (2), 49-80.

Zimmermann, Lisbeth, 2013: Too much translation to handle? The interaction between rule of law promotion and norm translation in post-conflict states. Paper prepared for the ISA Annual Convention 2013, San Francisco, 3.-6.4.2013, http://files.isanet.org/ConferenceArchive/944e135f333e4ec3b4f4cdf7da416128.pdf (Stand: 16.4.2013).

Zwingel, Susanne, 2012: How Do Norms Travel? Theorizing International Women's Rights in Transnational Perspective, in: International Studies Quarterly 56 (1), 115-129. 
Korrespondenzanschrift:

Elvira Rosert, M.A.

Goethe-Universität Frankfurt am Main

Fachbereich Gesellschaftswissenschaften

Institut für Politikwissenschaft

Campus Westend - PEG Gebäude

Hauspostfach 28

Grüneburgplatz 1

60323 Frankfurt am Main

E-Mail: rosert@soz.uni-frankfurt.de 